\title{
Ultrasound detection using polymer microring optical resonator
}

\author{
Shai Ashkenazi ${ }^{\text {a) }}$ \\ Department of Biomedical Engineering, University of Michigan, Ann Arbor, Michigan 48109 \\ Chung-Yen Chao and L. Jay Guo \\ Solid-State Electronics Laboratory, Department of Electrical Engineering and Computer Science, \\ University of Michigan, Ann Arbor, Michigan 48109 \\ Matthew O'Donnell \\ Department of Biomedical Engineering, University of Michigan, Ann Arbor, Michigan 48109
}

(Received 21 June 2004; accepted 18 October 2004)

\begin{abstract}
Application of polymer waveguide microring resonators for high-frequency ultrasound detection is presented. The device consists of a microring optical resonator coupled to a straight optical waveguide which serves as input and output ports. Acoustic waves irradiating the ring waveguide induce strain modifying the waveguide cross section. As a consequence, the effective refractive index of optical waves propagating along the ring is modified. The sharp wavelength dependence of the high $Q$-factor resonator enhances the optical response to acoustic strain. High sensitivity is demonstrated experimentally in detecting broadband ultrasound pulses from a $10 \mathrm{MHz}$ transducer. Methods of extending the technique to form multi-element ultrasonic arrays for imaging applications are proposed. (C) 2004 American Institute of Physics. [DOI: 10.1063/1.1829775]
\end{abstract}

Detection of ultrasound using optical techniques has gained increasing interest during recent years. Resonant optical structures such as etalons, fiber gratings and dielectric multilayer interference filters were employed as ultrasound sensing elements. ${ }^{1-4}$ These devices all rely on the interaction of an optical field confined in a resonance cavity with a transient ultrasonic field. The interaction takes place due to the modulation of the optical properties of the resonance cavity in response to the strain induced by the ultrasonic field. The sharp resonance line of the cavity amplifies the optical response to the transient strain. The advantage of using optical methods over conventional piezoelectric based sensors is apparent when small (micrometer scale) element size ultrasound arrays are considered. The effective area of an optically based ultrasound sensor is determined by the size of the confined optical field, which in most cases can be scaled down to micrometer size without increasing detection noise. For the case of a piezoelectric sensing element, however, noise increases with reduced element size.

It should be noted that similar principles have been applied in developing chemical and biological sensors utilizing integrated optics design. Microresonator sensors can rely on accurate measurement of the effective refractive index change of the guided mode inside waveguides, ${ }^{5}$ due to the presence of biomolecules on the surface of sensing areas or in the solution surrounding the devices. ${ }^{6-8}$ In microresonator sensors, signals can be detected by measuring resonance shifts, or alternatively, by measuring output intensity changes from the device at a fixed wavelength. The latter scheme is especially useful for detecting very small changes in the effective index, and we show that such a property can be used to detect, sensitively, the resonator's response to an incident ultrasound pulse. We propose this scheme for high-frequency ultrasound detection. Ultrasound imaging with high-spatial

\footnotetext{
a) Author to whom correspondence should be addressed; electronic mail:
} shaia@eecs.umich.edu resolution can be achieved using arrays of integrated microring resonators.

In this letter we show that high-frequency ultrasound receiver elements can be realized using a high $Q$-factor microring optical resonator. A microring resonator is typically designed as a closed-loop waveguide (the ring resonator) and a straight waveguide that couples light in and out of the resonator. The coupling is confined to a region where the light distributions at the two waveguides significantly overlap. Ultrasound waves stress the complex waveguide's structure deforming both the ring waveguide and the coupling region. These deformations induce a wavelength shift in the resonance structure. Amplified modulation of the transmitted light at a fixed wavelength can be obtained by choosing a wavelength at the steep edge of the transmission spectrum.

A polystyrene (PS) waveguide structure, consisting of a closed-loop, racetrack-shaped resonator coupled with a straight waveguide, was fabricated on a silicon substrate using an imprinting technique. ${ }^{9}$ The resonator diameter (of the two semicircles) is $60 \mu \mathrm{m}$ and its cross-section dimension is $2.3 \mu \mathrm{m} \times 1.8 \mu \mathrm{m}$. Figure 1 shows a scanning electron microscope (SEM) image of the device and the waveguide's cross section. The transmission spectrum was measured using a tunable laser (Santec TSL-220) spanning the range of

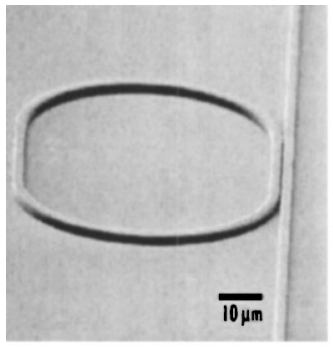

(a)

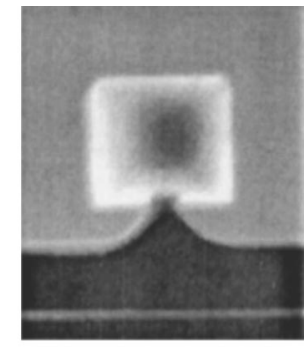

(b)
FIG. 1. SEM pictures of (a) perspective view and (b) cross-sectional view of polymer microring resonator fabricated by direct imprinting method. 


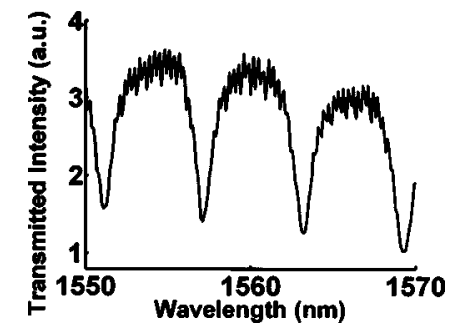

FIG. 2. Optical transmission spectrum of a microring device.

$1530-1610 \mathrm{~nm}$ at $1.5 \mathrm{~mW}$ output power. The polarization of the input beam was controlled using a half wave plate and a polarizer. The laser beam was coupled into the waveguide and collected by objective lenses. The measured transmission spectrum, using de-ionized water as cladding material, is shown in Fig. 2. The resonator has a moderate $Q$ of 1000 .

We tested the acoustic sensitivity of the device using a broadband ultrasonic transducer (Panametrics V311, $10 \mathrm{MHz}, 12.5 \mathrm{~mm}$ diameter, $19 \mathrm{~mm}$ focal length) to insonify the microring waveguide. A pulser/receiver (Panametrics 5072PR) drove the ultrasound transducer. The transducer was coupled to the microring resonator using a horn shaped acrylic coupler filled with water based solid gel (SeaKem LE Agarose 2\%, Cambrex BioSciences). The horn coupler was designed so that the focused acoustic radiation pattern is confined within the gel. This minimizes acoustic reflections from the acrylic walls of the coupler. The horn has an opening at the focal plane to couple acoustic radiation to the microring.

A shematic of the experimental setup is shown in Fig. 3. The ring resonator was immersed in a liquid ultrasound coupling gel (Aquasonic100, Parker Laboratories Inc.) which served both as an optical cladding of the microring waveguide and acoustical coupling to the opening of the horn coupler. The output wavelength of the tunable laser was set to a fixed wavelength near one of the resonance peaks $(\lambda$ $=1560.0 \mathrm{~nm}$ ) which was selected after performing a fine scan of the resonance transmission curve (see inset in Fig. 4). The output power was set to $1.5 \mathrm{~mW}$. A fast response amplified InGaAs photodiode (Newport 818-BB-30A) was used to measure the transient response of the optical transmission following acoustic excitation. The signal of the amplified photodiode was further amplified by the receiver within the pulser/receiver and recorded using a $400 \mathrm{MHz}$ digital sampling oscilloscope (Tektronix TDS360).

A modulation signal was clearly observed following an acoustic propagation delay time (Figs. 4(a)-4(c)). The signal to noise ratio of a single shot wave form was about 40 . A clear illustration for the wavelength shift effect of the ultrasonic wave is obtained by observing the different signal re-

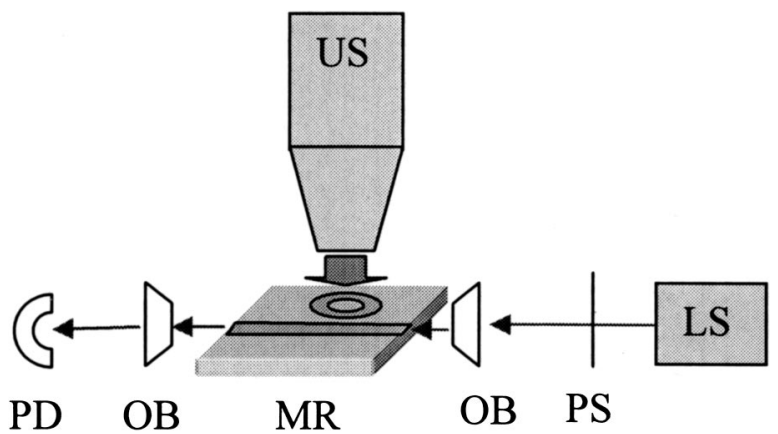

FIG. 3. Experimental setup used for measuring the detection sensitivity of a microring (MR) consisting of a tunable laser (LS), polarizing optics (PS), objective lenses (OB), photodetector (PD) and ultrasound pulse transmitter (US).

sponse at three different wavelengths of excitation. Two of the wavelengths are located at the opposite sides of the resonance peak [Figs. 4(a) and 4(c)] while the third wavelength [Fig. 4(b)] was set to be close to the resonance peak. The excitation wavelength, in each case, is marked as a vertical line on the inset of the corresponding figure. It is apparent that the signals corresponding to the two sides of the resonance peak have a linear response of opposite polarity, as expected due to sign change in the transmission slope. A dominant quadratic response is observed for the third signal 4(b) where the excitation wavelength corresponds to a minimum of the transmission spectrum (the resonance peak). The specific wavelength dependence of the response indicates that bulk effects, such as the mechanical displacement of the device under an ultrasound pulse, are not significant.

To evaluate the acoustic sensitivity of the device, we measured the acoustic output using a calibrated membrane polymer polyvinylidene fluoride hydrophone (GEC-Marconi Y-33-7611). The acoustic output was measured at the tip of the horn coupler within the focal zone of the acoustic radiation. The peak pressure at the maximal point was $64 \mathrm{KPa}$. The modulation of the optical transmission is assessed by normalizing the recorded wave form by the overall voltage gain and transimpedance gain, yielding a modulated optical power of $8.0 \mu \mathrm{W}$. Normalizing the modulation transmission power by the mean optical power reaching the detector gives a modulation depth of $\operatorname{Tr}=0.2$. The measured sensitivity is therefore $D=\operatorname{Tr} / \mathrm{Pa}=3 \times 10^{-3} \mathrm{kPa}^{-1}$. This value is much higher than the expected sensitivity due to straining of the microring waveguide, suggesting that different mechanisms may be responsible for the high sensitivity observed.

To conclude, we have demonstrated the application of an optical microring resonator as a small (less than one acoustic wavelength) single element ultrasound receiver. The experi-
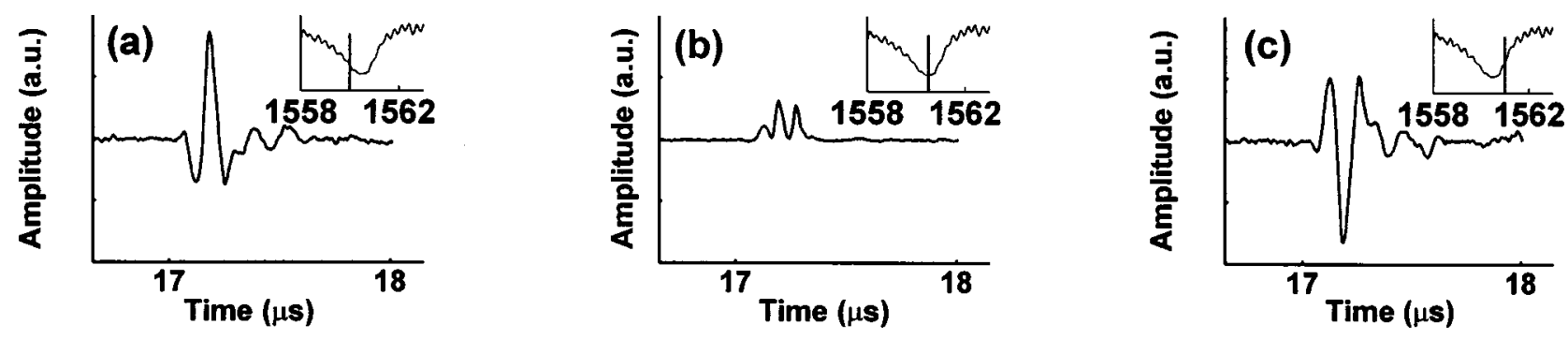

FIG. 4. Ultrasound modulated transmission signals measured at (a) $\lambda=1560.0 \mathrm{~nm}$, (b) $\lambda=1560.5 \mathrm{~nm}$, and (c) $\lambda=1561.0 \mathrm{~nm}$. Insets show detailed transmission spectrum. A vertical line in each spectrum indicates the corresponding excitation wavelength for each modulation signal. 
mental characterization of the microring ultrasonic receiver shows high sensitivity and high bandwidth exceeding $10 \mathrm{MHz}$. The technique can be extended to realize a multielement array ultrasonic receiver, using known techniques in the field of optical communication. Arrays of microring resonators were demonstrated to operate as add-drop filter arrays for telecommunication applications, ${ }^{10}$ where multiple microring resonators (eight elements) are coupled to a common input bus waveguide and each has its own output waveguide. In this device, the ring radii were changed with a fixed increment between each element and therefore each resonator has a different resonance peak location. Coupling the light in the common bus waveguide to a specific output waveguide is achieved by choosing the appropriate wavelength. The same mechanism is applicable to the ultrasound receiver array where each element is addressed by selecting the appropriate wavelength at the input port. Separate output waveguides for each element are not required in this case since the common bus waveguide can serve both as input and output ports. A two-dimensional (2D) array of $N \times M$ elements can be realized using $N$ rows, where each row has $M$ microring elements and its own bus waveguide.

The design of a one-dimensional or 2D ultrasonic array using integrated optics device techniques offers unique advantages such as ruggedness, small size, rf immunity and low manufacturing cost, which could be beneficial in various ultrasound applications. A particularly appealing medical application is the design of a small integrated optical device which will operate as an intravascular imaging probe. The ultrasound pulse generator could be integrated using photoacoustic methods, ${ }^{11}$ therefore eliminating the need for any electrical cabling since fiber optics carry both input and output signals. The high element density required for high resolution intravascular imaging dictates an upper limit of $20 \mu \mathrm{m}$ on the ring diameter. Reducing the size of the rings will also increase the free spectral range and therefore will increase the number of elements that can share a common bus waveguide. The drawback of small size rings is increased radiation loss, that may reduce the resonance $Q$-factor and therefore decrease sensitivity. Further study is required to address these issues.

${ }^{1}$ J. D. Hamilton, T. Buma, M. Spisar, and M. O'Donnell, IEEE Trans. Ultrason. Ferroelectr. Freq. Control 47, 160 (2000).

${ }^{2}$ P. C. Beard, F. Perenncs, and T. N. Mills, IEEE Trans. Ultrason. Ferroelectr. Freq. Control 46, 1575 (1999).

${ }^{3}$ J. G. Laufer, P. C. Beard, and T. N. Mills, Rev. Sci. Instrum. 73, 3345 (2002).

${ }^{4}$ V. Wilkens and Ch. Koch, Opt. Lett. 24, 1026 (1999).

${ }^{5}$ B. E. Little, S. T. Chu, and H. A. Haus, Opt. Lett. 23, 1570 (1998).

${ }^{6}$ E. Krioukov, D. J. W. Klunder, A. Driessen, J. Greve, and C. Otto, Opt. Lett. 27, 512 (2002).

${ }^{7}$ E. Krioukov, D. J. W. Klunder, A. Driessen, J. Greve, and C. Otto, Opt. Lett. 27, 1504 (2002).

${ }^{8}$ C. Y. Chao and L. J. Guo, Appl. Phys. Lett. 83, 1527 (2003).

${ }^{9}$ C. Y. Chao and L. J. Guo, J. Vac. Sci. Technol. B 20, 2862 (2002).

${ }^{10}$ S. Suzuki, Y. Hatakeyama, Y. Kokubun, and S. T. Chu, J. Lightwave Technol. 20, 745 (2002).

${ }^{11}$ T. Buma, M. Spisar, and M. O'Donnell, IEEE Trans. Ultrason. Ferroelectr. Freq. Control 50, 1065 (2003). 